\title{
ANÁlisis DE LA TRADUCCIÓN A LA VISTA EN EL ÁMBito JURÍdico CASTELLANO-CHINO Y LA APLICACIÓN DE LA LINGÜÍSTICA FORENSE. / AN
} ANALYSIS OF THE SIGHT TRANSLATION IN SPANISH-CHINESE AND APPLICABILITY OF FORENSIC LINGUISTICS

\author{
Tian Xiaolong \\ Universidad de Alcalá, España \\ xiaolong.tian@edu.uah.es
}

\begin{abstract}
Resumen: Hoy en día hay cada vez más inmigrantes chinos en España. Como trabajan y viven en este país, a veces tienen que enfrentarse a algunas dificultades jurídicas. Por esta razón acuden a pedir ayuda a los traductores e intérpretes con el fin de afrontar las dificultades mencionadas. No obstante, es difícil distinguir a los traductores chinos formados de los que no tienen formación específica, lo que hace que los usuarios y los clientes estén descontentos con lo que han traducido o han interpretado los traductores e intérpretes. Sabemos que son múltiples las razones de este fenómeno. Por lo tanto, analizaremos este fenómeno desde el aspecto de la lingüística forense aplicada a la traducción a la vista en el ámbito jurídico castellano-chino con el fin de mejorar la calidad del trabajo realizado por los traductores e intérpretes. Esperamos que este artículo pueda favorecer la traducción a la vista en el ámbito jurídico castellano-chino. Para realizar este análisis, hemos buscado dos documentos jurídicos que se han traducido a la vista por intérpretes. De este modo podremos conseguir materiales comparables con el fin de obtener más datos para el análisis. También hemos realizado una serie de encuestas con preguntas tanto objetivas como subjetivas para determinar los problemas y dificultades encontradas durante la traducción a la vista. El objetivo es poder encontrar un método adecuado para reducir y seleccionar los conflictos lingüísticos, los problemas de lógica lingüística y de estructura del lenguaje.
\end{abstract}

Palabras clave: Lingüística forense; Traducción a la vista; Chino-castellano; Ámbito jurídico.

\footnotetext{
Abstract: The Taipei Mass Rapid Transit System (Taipei MRT) began operations in 1996 with the technical support of its French contractor MATRA Transport. The system now consists of five lines. Expected to further enhance the public transportation of the capital city, the Songshan Line, the latest addition of the Taipei MRT, was launched in November 2014 and takes passengers to
} 
the Songshan Airport. As it started service, the Taipei MRT brings more convenience to the public and becomes an internationalized transportation network. The Taipei MRT Guide (hereinafter referred to as "the Guide") is available in 15 languages. For the public service in Taiwan, it is the most internationalized among all brochures published by governments. This study intends to analyze the multi-language support of the public services in Taiwan. In addition, it examines the translations of the Taipei MRT Guide, which is considered the most successful translations in the category of public service so far. By comparing the Chinese original and the Spanish version of the Guide and discussing the Spanish translation, it evaluates the practical aspect of the Spanish version and Spanish native speakers' acceptance towards the Spanish translation.

Keywords: Public services; Rapid transit system; Taiwan; Taipei; Zero translation.

\begin{abstract}
摘要：台灣捷運從 1996 年通車，得力於法國公司馬特拉(MATRA)提供技術上的協助。目 前台北共計五條捷運線, 2014 年 11 月才通車的松山新店線可連接至松山機場, 其目標為改 善首都交通，並提供舒適與國際化的交通網絡。「台北捷運營運資訊簡介」有 15 種語言版本 ，是台灣公共服務領域中最國際化的官方文本。本研究目的為分析台灣公共服務的多語背 景，探討捷運文本翻譯，亦即目前最成功的公共服務筆譯類別。透過中文和西文版本的比較 、探討西班牙語翻譯，評估其實用性及西語為母語者的接受度。
\end{abstract}

關鍵字: 公共服務; 捷運; 台灣; 台北; 零翻譯.

\title{
1. Introducción al desarrollo de la lingüística forense en China
}

Hoy en día, China ha logrado muchos éxitos en el desarrollo tanto económico como político y también en las investigaciones de lingüística forense y de traducción e interpretación jurídicas. No obstante, dichas investigaciones se han retrasado, comparadas con las de los países occidentales, debido a las siguientes causas: la dificultad del dominio de los dos idiomas (el chino y la otra lengua del trabajo), la carencia del intercambio de los logros y experiencias científicos, y la escasez de la documentación relativa a la lingüística forense y a la traducción e interpretación jurídicas, etc. A continuación, nos centraremos en el desarrollo de la lingüística forense en China.

Es un ámbito nuevo, no sólo en la lingüística aplicada, sino también en la jurisprudencia, y por lo tanto se trata de un campo interdisciplinario. Desde los años sesenta, los abogados y ministros buscaron auxilio en los lingüistas dado que habían encontrado algunas dificultades en el idioma y el lenguaje en aspectos concretos como la investigación del crimen, el enjuiciamiento, etc. En China existen muchas naciones, y los dialectos e idiomas de algunas de ellas son muy difíciles de comprender. Por lo tanto, el desarrollo de la investigación de la lingüística forense favorecería la legislación de China para los ciudadanos de las etnias minoritarias chinas.

En los años noventa, algunos investigadores chinos introdujeron nuevos estudios sobre este campo basado en los ya realizados por países occidentales, sembrando el germen de la investigación lingüística forense en China. En 1994, un lingüista chino, Wu Weiping, escribió en chino un artículo titulado "Lingüística forense: congreso, asociación y publicación" 
en una publicación llamada Lingüística extranjera. En él introdujo, por primera vez, a los lectores chinos la ciencia denominada forensic linguistics (en español: "lingüística forense") y el estudio relativo a ésta. Este término científico fue traducido por el escritor como 法律语 言学 (literalmente en español: "lingüística del derecho", sinónimo de "lingüística forense").

En algunas universidades tanto de Pekín como de Shanghái la docencia de lingüística forense se inició en 2003. No obstante, la universidad que ofertó esta carrera por primera vez fue la Universidad de Estudios Extranjeros de Guangdong. Es decir, el sector universitario consideró que la formación de los alumnos universitarios en lingüística forense era importante e imprescindible.

\section{Objetivos generales de la presente investigación}

Si no existiese una meta o un objetivo hipotético en una investigación y simplemente se narrase lo que sucede dentro de dicha investigación, ese tipo de estudio no sería válido. Por ello, los objetivos generales de la presente investigación son:

1. Mejorar la calidad de la traducción a la vista de castellano-chino en el ámbito jurídico.

2. Profesionalizar y perfeccionar la enseñanza de la traducción a la vista de castellano-chino en el ámbito jurídico.

3. Facilitar y favorecer la utilización de la estrategia traductológica durante el proceso de la traducción a la vista.

De acuerdo con la página web principal de International Association of Forensic Linguists (IAFL, Asociación Internacional de Lingüistas Forenses), los objetivos de la lingüística forense son:

- Study of the language of the law, including the language of legal documents and of the courts, the police, and prisons. (El estudio del lenguaje jurídico, incluyendo el lenguaje de documentos jurídicos, de los tribunales, de la policía y la prisión).

- The use of linguistic evidence (phonological, morpho-syntactic, discourse-pragmatic) in the analysis of authorship and plagiarism, speaker identification and voice comparison, confessions, linguistic profiling, suicide notes, consumer product warnings. (El uso de la evidencia lingüística —fonológica, morfosintáctica, discurso-pragmática — en el análisis de la autoría y el plagio, la identificación del hablante y la comparación de voz, las confesiones, los perfiles lingüísticos, las notas de suicidio, las advertencias de productos de consumo).

- The use of language as evidence in civil cases (trademark, contract disputes, defamation, product liability, deceptive trade practices, copyright infringement). (El uso del lenguaje como prueba en causas civiles — marca registrada, disputas de contratos, difamación, responsabilidad productiva, prácticas comerciales engañosas, infracción de derechos de autor).

- $\quad$ The alleviation of language-based inequality and disadvantage in the legal system. (La reducción de la desigualdad y las desventajas basadas en el lenguaje y en el sistema jurídico).

- The interchange of ideas and information between the legal and linguistic 
communities (El intercambio de ideas e información entre las comunidades jurídicas y lingüísticas).

- Research into the practice, improvement, and ethics of expert testimony and the presentation of linguistic evidence, as well as legal interpreting and translation; (La investigación de las prácticas, la mejora y la ética de los testimonios de expertos y la presentación de la evidencia lingüística, así como la interpretación y traducción jurídica).

- Better public understanding of the interaction between language and the law.(Una mejor comprensión pública de la interacción entre el lenguaje y la ley).

Por lo tanto, podemos decir que los objetivos de la presente investigación se corresponden con algunos objetivos de IAFL, ya que ambos forman parte del estudio de lingüística forense.

\section{Teoría y metodología aplicada en esta investigación}

Como hemos visto hasta ahora, la lingüística forense ha influido en la traducción a la vista de castellano-chino en el ámbito jurídico. Por lo tanto, las teorías de dichos aspectos (lingüística forense y traductología) pueden aplicarse conjuntamente. A continuación, analizaremos diferentes partes teóricas.

De acuerdo con Peter Trudgill (1976), la lingüística forense pertenece al contexto de la lingüística aplicada. Sabemos que, hoy en día, en los círculos académicos existe un debate sobre la pertenencia académica de la linguiística forense. Según su opinión, dentro del contexto de la lingüística aplicada, la lingüística forense es una ciencia interdisciplinaria que conecta la investigación lingüística-sociolingüística con los distintos problemas sociales relacionados con el derecho, tales como el análisis del lenguaje, la investigación dialectal, las variaciones lingüísticas, la estilística, etc.

Algunos investigadores, consideran, desde el punto de vista del ámbito lingüístico, que la lingüística forense pertenece a la lingüística aplicada, puesto que soluciona el uso problemático sociolingüístico. Sin embargo, la metodología de las investigaciones sobre la relación entre el derecho y el lenguaje que han realizado los investigadores referidos, tales como Liao Meizhen (2006) y David Millinkoff (1963), se basaba en señalar la verdad y la función del derecho de acuerdo con la verdad del lenguaje. El representante de estos investigadores es David Mellinkoff, quien expresó claramente esta opinión en su obra titulada The Language of the Law en 1963. Al comienzo del estudio sobre la lingüística forense las afirmaciones eran fundadas, ya que en aquel entonces dichas investigaciones solían ser superficiales. Descubrieron que la lingüística forense estaba basada en la lingüística. No obstante, dichos investigadores no tenían conocimientos de derecho ni participaban en las prácticas sobre la aplicación del derecho, sino que simplemente podían conjeturar sobre el contexto que surgiría para investigar el lenguaje del derecho. Este fenómeno ha segregado la teoría lingüística forense de su práctica. Por lo tanto, esta opinión es ahora discutible, es decir, no es correcta, si bien tiene sentido.

Por el contrario, otros investigadores creen que, desde el punto de vista del objetivo de esta ciencia, se trata de una disciplina jurídica, ya que lo que estudia e investiga tiene que 
ver con lo jurídico. En la obra titulada Language and the law de John Gibbons (1994) se mostraba la relación estrecha entre la lingüística forense, el lenguaje y la ley: "Language constructing law". También afirma en la misma obra que "Law is language [...] Laws are coded in language, and the processes of law are through language". Es decir, todo el sistema jurídico, tanto las leyes como los procesos jurídicos, tienen que existir y efectuarse a través del lenguaje. El estudio de este tipo de lenguaje y la relación entre el lenguaje y el derecho se han convertido en la lingüística forense. John Gibbons (1994) afirma que la lingüística forense incluye todos los aspectos del ámbito jurídico y lingüístico, e incluso de la traducción jurídica y la interpretación jurídica, así como de la traducción a la vista en el ámbito jurídico (según IAFL). En los párrafos anteriores expuse que la linguiística forense incluía la investigación de la interpretación y la traducción jurídica, lo que da una base teórica a la presente investigación.

La lingüística forense, como el propio nombre indica, es una ciencia interdisciplinaria. El primer término, "lingüística", hace referencia a un estudio científico sobre el lenguaje natural o el lenguaje humano, pudiéndose dividir en diversas ramas interdisciplinarias de la lingüística tales como la adquisición del lenguaje, la lingüística aplicada, la sociolingüística, la psicolingüística, etc. Entre ellas, la lingüística forense es un estudio que pertenece a la lingüística aplicada puesto que es un aspecto que se centra en los problemas de la sociedad relacionados con el uso del lenguaje. Por ello, en nuestro opinión, la lingüística forense pertenece a la lingüística aplicada, puesto que sirve para gestionar el uso problemático del lenguaje jurídico, y dicho lenguaje es un tipo de lenguaje específico.

Siguiendo con la teoría traductológica, ahora veremos la teoría de la traducción semántica y comunicativa de Peter Nermark (1988). Durante el proceso de la traducción en los servicios públicos (SSPP), tanto de la traducción como de la interpretación e incluso de la traducción a la vista, se aplica más la traducción semántica (o sea, traducción literal) que la traducción comunicativa. El contexto, en la traducción a la vista en el ámbito jurídico castellano-chino, se centra en los documentos jurídicos. Puesto que la traducción jurídica y también el uso del lenguaje jurídico requieren exactitud durante el proceso de la producción de la traducción, la traducción libre, o traducción comunicativa, ha sido una estrategia traductológica que no se suele utilizar en la traducción a la vista en el ámbito jurídico.

Por último, expondremos la teoría de la equivalencia de Eugene Nida (1969). Se trata de la equivalencia dinámica y formal, en la que se abarca no sólo la equivalencia del contenido de la traducción, sino también la forma de la traducción. Aunque su teoría se aplicó en principio a la traducción de la Biblia, en realidad, esta equivalencia se podría aplicar a diversos ámbitos de la traducción (traducción literal, comercial, en los SSPP, etc.) y en sus diversas modalidades (traducción, interpretación, traducción a la vista, etc.).

\section{Metodología de la presente investigación}

Hemos elegido dos textos jurídicos en español con el fin de que los traductores profesionales y estudiantes universitarios chinos de la carrera de traducción realicen la traducción a la vista, descubriendo las aplicaciones de la lingüística forense y los problemas y errores encontrados en sus traducciones. Además, hemos agrupado a los participantes en dos grupos: Grupo 1 (traductores profesionales, 10 personas) y Grupo 2 (estudiantes universitarios, 10 personas). 
También hemos conseguido grabaciones como los materiales comparativos entre dos grupos con el fin de obtener más datos para el análisis y observar la influencia de la lingüística forense en la calidad de la traducción a la vista. Es decir, hemos empleado una metodología cuantitativa para procesar la investigación. Hemos conseguido un total de 20 participantes sobre la traducción a la vista de los dos documentos jurídicos: uno es un atestado (Texto 1 del Apéndice) y el otro es la diligencia de información de derechos al detenido (Texto 2 del Apéndice).

A continuación podemos ver y analizar los datos de los participantes (Tabla 1):

\begin{tabular}{|l|c|c|c|c|}
\hline Género & Española & Porcentaje & China & Porcentaje \\
\hline Hombre & 2 & $10 \%$ & 5 & $25 \%$ \\
\hline Mujer & 2 & $10 \%$ & 11 & $55 \%$ \\
& & & & \\
\hline
\end{tabular}

Tabla 1. Distribución de nacionalidad

De de los 20 participantes, además de cuatro españoles, hay cuatro chinos que tienen el diploma de DELE B2 (Intermedio) o el nivel equivalente a B2. De de todos los integrantes, trece son mujeres y los demás son hombres. Existen seis participantes que han obtenido el nivel equivalente al nivel DELE C1 (Avanzado). Otros seis participantes tienen el diploma de DELE C2 (Superior) o un diploma equivalente a este nivel, de los cuales cinco mujeres y solo un hombre. Véase la Tabla 2 (Distribución del nivel de español).

\begin{tabular}{|l|c|c|c|c|}
\hline $\begin{array}{c}\text { Nivel de español } \\
\text { Género }\end{array}$ & $\begin{array}{c}\text { DELE B2 } \\
\text { (Intermedio) }\end{array}$ & $\begin{array}{c}\text { DELE C1 } \\
\text { (Avanzado) }\end{array}$ & $\begin{array}{c}\text { DELE C2 } \\
\text { (Superior) }\end{array}$ & NATIVO \\
\hline Hombre & 2 & 2 & 1 & 2 \\
\hline Mujer & 2 & 4 & 5 & 2 \\
\hline
\end{tabular}

Tabla 2. Distribución del nivel de español

\begin{tabular}{|c|c|c|c|c|}
\hline Profesión & Estudi & iversitarios & Tra & ores \\
\hline Género & Grado & $\begin{array}{c}\text { Máster } \\
\text { Universitario }\end{array}$ & Profesores & Intérpretes \\
\hline Hombres & 1 & 3 & 1 & 2 \\
\hline Mujeres & 2 & 4 & 4 & 3 \\
\hline
\end{tabular}

de profesión

En cuanto a la profesión de los participantes, diez de ellos son estudiantes tanto de grado (3) como de máster de la traducción e interpretación (7), cuatro hombres y seis mujeres. 
El resto son expertos y profesionales de la traducción e interpretación, tales como profesores (5) e intérpretes profesionales (5). En este grupo, hay tres hombres y siete mujeres. Véase la Tabla 3 (Distribución de profesión).

\section{Análisis de los textos elegidos}

En esta sección, empezaremos a analizar las muestras que obtenidas sobre la traducción a la vista desde el punto de vista de la lingüística forense. Considerando las limitaciones en cuanto al número de páginas de este artículo, hemos seleccionado algunos ejemplos típicos y los hemos analizado con el fin de obtener resultados específicos y extraer conclusiones.

En general, los participantes han realizado este trabajo con esfuerzo. Los traductores profesionales lo han terminado con precisión y perfección; sin embargo, los alumnos universitarios han cometido algunos errores y han surgido algunos problemas. A continuación, los enumeraremos y trataremos más detalladamente.

\subsection{Sobre las características del lenguaje forense}

El lenguaje forense tiene características tales como neutralidad, exactitud, estandarización y uniformidad. Es difícil para un traductor profesional producir una traducción que corresponda a las características anteriores durante la traducción a la vista, ya que ésta debe realizarse en un tiempo corto, por lo que no tiene tanto tiempo para reordenar la oración. Veamos aquí un ejemplo del texto 1:

(1) Que no tiene/n más que decir, firmando su declaración en prueba de conformidad, en unión del Instructor. CONSTE Y CERTIFICO.

La versión traducida por un profesional del Grupo 1:

别无他事, 报案人在他所陈述的情况之下签名并作为证据, 警长在旁边签字。书记员记 录并对此证明。

La versión traducida por un alumno del Grupo 2:

由于没有别的事情, 双方在下方签名, 警长在下面签字。我记录并证明此事。

Veamos otra versión que realizó otro alumno del Grupo 2:

因为没有别的事情, 报案人与警长签字, 我记录并在此证明。

Arriba se exponen una serie de datos del Grupo 1 y 2 que serán comparados a continuación. Concretamente, al comparar con la traducción realizada por los participantes del Grupo 2, la versión del Grupo 1 es más completa, precisa y neutra ya que en la versión del Grupo 2 se ha empleado la palabra «我» (en castellano: yo). Como el texto jurídico se caracteriza por la autonomía, la aplicación del pronombre personal, no se ha mostrado la neutralidad del lenguaje forense. Además, se ha utilizado la frase «由于没有别的事情》 (en castellano: Como no tiene otros asuntos). De este modo, la otra versión parece como si fuera una traducción muy breve, puesto que omite algunos datos como "en prueba de conformidad", 
así como la aplicación de la primera porción de toda la oración 因为没有别的事情, como si fuese el lenguaje coloquial en lugar del lenguaje jurídico.

Veamos otro ejemplo sobre esto en el texto 1:

(2) Que ha sido informado de la obligación legal que tiene de decir la verdad (Art.433 de L.E.Cr.) y de la posible responsabilidad penal en la que puede incurrir en caso de acusar o imputar falsamente a una persona una infracción penal o con temerario desprecio hacia la verdad (Art. 456 de Código Penal), simular ser responsable o víctima de una infracción penal (Art.457 de Código Penal), o faltar a la verdad en su testimonio (Art.458 de Código Penal).

La versión traducida por el Grupo 1:

报案人被告知陈述事实是法律义务 (刑事诉访法第 433 条), 虚假指控、错误给人定罪 或罔顾真相 (刑法典第456 条)、假装是犯罪嫌疑人或受害者（刑法典第457 条）或在 证据中缺少事实依据 (刑法典第458 条) 都将负刑事责任。

La versión traducida por el Grupo 2:

报案人被知会陈述事实是义务（L.E.Cr. 第 433 条），错误指控或误判或不顾真相（刑法 第456 条)、装作是犯罪嫌疑人或受害者（刑法第457 条）或在证据中缺少依据（刑法 第458 条) 都将负刑事责任。

Hemos analizado las dos traducciones y hemos visto que los dos párrafos traducidos son comprensibles. Desde este punto de vista, las dos traducciones se han realizado perfectamente. O sea, se ha cumplido un requisito básico en cuanto a la traducción, la comprensibilidad. Con respecto a otro requisito específico de la traducción, la exactitud, la del Grupo 1 es mejor que la del Grupo 2. Además, en la traducción del Grupo 2 las palabras subrayadas son traducciones inadecuadas a causa de la carencia de la terminología del derecho en español y del mal uso del lenguaje; e incluso la falta de traducción de algunos conceptos importantes como "L.E.Cr."(Ley de Enjuiciamiento Criminal)

\subsection{Sobre la terminología}

De acuerdo con nuestro análisis, hemos visto que algunos profesionales y casi todos los alumnos universitarios no distinguen entre los conceptos "término" y "lenguaje profesional". Generalmente, los términos son unas palabras o un conjunto de palabras de un ámbito específico. Sin embargo, el lenguaje profesional es una forma de la aplicación del idioma dentro de cierto grupo de personas (jueces o abogados) o una cierta circunstancia (en el tribunal). Pueden ser una serie de oraciones. A continuación, veamos las diferencias entre estos conceptos.

Término: Conjunto de las voces técnicas propias de una facultad.

Lenguaje profesional: Un lenguaje específico que se suele utilizar dentro de un cierto grupo de personas. Si no se recibe formación en la aplicación de este lenguaje, es muy difícil entenderlo.

Para mejorar nuestra comprensión sobre los dos conceptos, incluimos algunos ejemplos para ver las diferencias entre ellos: 
(3) Leída y conforme. La firma, de lo que como Secretario CERTIFICO. (La última oración del texto 2) 本人已阅读并确认签字，书记员特此证明。

Es lenguaje profesional, o sea, lenguaje jurídico, ya que se trata de una serie o un conjunto de oraciones que se incluyen al final de los textos jurídicos con el fin de terminar dicho texto jurídico. Es una sección imprescindible e importante, es decir, un formalismo para completar un texto jurídico, no una o unas palabras técnicas. A continuación, veamos otro ejemplo:

\section{(4) Un presunto delito. 推定罪行}

Obviamente, podemos ver que es una expresión del vocabulario técnico: presunto y delito. Aquí un es un artículo, pero determina la cantidad y el sexo de la palabra presunto. Las dos palabras técnicas son del ámbito jurídico y se suelen utilizar para este aspecto. Por ello, estas palabras, o sea, este sintagma es un término, no es lenguaje jurídico.

\subsection{Sobre la facilidad de comprensión}

De acuerdo con los datos de análisis, hemos observado que la legibilidad de la versión del Grupo 1 es mejor que la del Grupo 2. Respecto a la facilidad de comprensión, algunos miembros del Grupo 1 no la realizaron correctamente, ya que su versión contiene muchos términos castellanos traducidos a términos chinos, lo que provoca la dificultad de comprensión.

(5) No declarar si no quiere, no declarar contra sí mismo y no confesarse culpable, y no contestar alguna/s de las preguntas que se le formulen o manifestar que sólo declarará ante el juez. (Texto 2)

Traducción de Grupo 1：如果犯罪嫌疑人不愿意，可不交代问题，不自证其罪，不 认罪，不回答警方提出的那些只有在法庭上才能回答的问题。

Traducción de Grupo 2: 如果犯罪嫌疑人不想回答，可不回答，不证明他的罪行， 不认罪，不回答警方提出的那些在法庭上的问题。

Analicemos este ejemplo. La traducción del Grupo 1 es apropiada y es difícil encontrar errores tanto estructurales y gramaticales como terminológicos y lingüísticos. Sin embargo, en la traducción del Grupo 2, podemos ver en las unidades subrayadas que sus traducciones son un poco difíciles de entender: 可不回答 (En castellano: No es necesario responder. ¿Qué no quiere responder?).

Finalmente, cabe destacar que aunque algunos alumnos del Grupo 2 también hayan tenido problemas con la facilidad de entendimiento a causa del uso inadecuado de la estrategia traductológica, el resto de los alumnos tradujeron los fragmentos utilizando oraciones y palabras sencillas para que los usuarios entiendan mejor el mensaje. Sin embargo, no se han tenido en cuenta otros elementos como la neutralidad, la exactitud y la uniformidad del lenguaje jurídico. 


\section{Conclusiones}

A raíz de la investigación y los análisis realizados, hemos obtenido las siguientes conclusiones:

- Durante el proceso de realización de la traducción a la vista, los estudiantes universitarios suelen cometer errores terminológicos, mientras que en el caso de los profesionales este tipo de errores es mucho menos frecuente.

- Algunos traductores y la mayoría de los alumnos no han transmitido la información de manera accesible para el usuario. A pesar de haber traducido los términos de castellano al chino, a veces algunas oraciones o palabras no se han traducido con exactitud. Esto se debe a la falta de formación o de conocimientos específicos de la lingüística forense y del derecho. Además, aunque hayan seguido el principio de equilibrio entre equivalencia dinámica y formal de Eugene Nida, no se tiene en cuenta la comprensión por los usuarios. Por último, una serie de errores se deben al uso inadecuado de algunas estrategias de traducción y a la falta de conocimiento de las características del lenguaje forense.

- Los alumnos universitarios no tienen muchos conocimientos sobre las características de la legislación en general y de las leyes españolas y chinas en particular. Por ello, aunque a veces entienden lo que dice la ley en castellano, no son capaces de transmitirlo en chino, por lo que necesitan mucha más práctica sobre este aspecto. También deben recibir una formación o disponer del conocimiento sobre derecho de ambos países. Este problema, aunque no tiene nada que ver con la lingüística forense, es un problema importante que decide la calidad de la traducción a la vista realizada.

\section{Recomendaciones}

Los alumnos universitarios necesitan más práctica durante la formación. Asimismo, tienen que consultar durante la formación en traducción artículos relacionados con la lingüística forense para mejorar la calidad de la traducción a la vista en el ámbito jurídico castellano-chino. Desde el punto de vista terminológico, deben hacer un glosario jurídico con el fin de dominar bien la terminología jurídica en español-chino, además de leer libros específicos para adquirir conocimientos sobre el ámbito jurídico. Además, no sólo deben mejorar el uso del español a fin de reducir el número de errores gramaticales que puedan cometer, sino también saber analizar la unidad de la traducción y utilizar adecuadamente las diferentes estrategias traductológicas.

\section{Referencias bibliográficas}

International Association of Forensic Linguists (IAFL). Purpose \& Aims y Further Aims. [Disponible en: http://www.iafl.org/]

Corsellis, A. 2010. Traduccion e interpretación en los servicios públicos. Granada: Comares. Gibbons, J. P. 2003. Forensic Linguistics: An Introduction to Language in the Justice System. 
Inglaterra: Blackwell Publishing.

Hurtado Albir, A. 2001. Traducción y traductología. Introducción a la traductología. Madrid: Cátedra.

Gómez Torrego, L. 2007. Análisis Sintáctico Teoría y Prácticas. Madrid: Grupo Editorial SM Internacional.

Llácer Llorca, E. V. 2004. Sobre la traducción: Ideas tradicionales y teorías contemporáneas. Valencia: Universidad de Valencia.

López Moreno, M. 1985. Introducción a la Interpretación. Granada: Comares.

Navarro Domínguez, F. (ed.) 2002. Introducción a la teoría y práctica de la traducción. Ámbito hispanofrancés. $2^{\mathrm{a}}$ edición. Barcelona: PPU.

Ordóñez Solís, D. 2002. "Lenguaje judicial: argumentación y estilo". Ley $n .{ }^{o}$ 5564. 12 de junio de 2002. Madrid: 1-10.

Orozco, M. y Sánchez Gijón, P. "La ontología como instrumento de información al servicio del traductor jurídico". Papers Lextra, 2.

Pasquau Liaño, M. 1996. "Las peculiaridades del lenguaje jurídico desde la perspectiva del jurista”. San Ginés Aguilar, P. y Ortega Arjonilla, E. (eds.). Introducción a la traducción jurídica y jurada (Francés-Español). Granada: Comares: 9-23.

Ramírez Bellerín, L. 1999. Del carácter al contexto: teoría y práctica de la traducción del chino moderno. Barcelona. Universitat Autònoma de Barcelona.

Terral, F. 2003. "Derecho comparado y traducción jurídica: relación de interdependencia". Sendebar, 14: 97-106.

Torres del Rey, J. 2003. "Tecnología y enseñanza de la traducción: panorama investigador, enfoque humanístico". García Peinado, M. Á. y Ortega Arjonilla, E. (dirs.) Panorama actual de la investigación en traducción e interpretación. 2 (2). Granada: Atrio: 343-359.

\section{Apéndice}

Texto 1

ATESTADO

Instructor: 79691

Secretario: 107513

报案表

报案表: 2073/12

警长编号:79691

书记警员: 107513
Atestado: 2073/12

Atestado $n^{o}: 2073 / 12$

Dependencia: SEVILLA-

COMISARIA LOCAL

-En SEVILLA, siendo las 13 horas 23 minutos del día 24 de enero de 2012, ante el Instructor y Secretario arriba mencionados.

-2012 年 1 月 24 日 13 点 23 分, 上述警员在塞维利亚受理此案。 
-COMPARECE: En calidad de DENUNCIANTE, quien mediante permiso de residencia $n^{\circ} \mathrm{Y}$ 0739972C, acredita ser YUBIN WANG, país de nacionalidad CHINA, varón, nacido en CHINA, el día 25/07/1989, hijo de YONGLE y XIANGFEI , con domicilio en C/CALDERÓN DE LA BARCA, 4, 8C, de ALCALÁ DE HENARES (MADRID), teléfono 603306003, y:

-报案人证明: 报案人姓名为王宇斌, 居留号为 Y0739972C, 中国人, 男, 出生日期为 1989 年 7 月 25 日, 父母为永乐及祥菲, 现居住在塞维利亚利贝尔街的 4 号 8C, 电话 号码为 603306003 。

MANIFIESTA: Que denuncia los hechos, que se detallan a continuación ocurridos entre el día 04/01/2012 y el día 20/01/2012, en Piso, CALDERÓN DE LA BARCA, 4, 8C, de ALCALA DE HENARES (MADRID).

陈述如下: 居住在塞维利亚利贝尔街的 4 号 $8 \mathrm{C}$ 的报案人举报从 2010 年 1 月 4 日至 2010 年 1 月 20 日发生的情况。

-Que ha sido informado de la obligación legal que tiene de decir la verdad (Art.433 de L.E.Cr.) y de la posible responsabilidad penal en la que puede incurrir en caso de acusar o imputar falsamente a una persona una infracción penal o con temerario desprecio hacia la verdad (Art. 456 de Código Penal), simular ser responsable o víctima de una infracción penal (Art.457 de Código Penal), o faltar a la verdad en su testimonio (Art.458 de Código Penal).

报案人被告知陈述事实是法律义务 (刑事诉讼法第 433 条), 虚假指控或错误给人定罪 或罔顾真相（刑法典第 456 条）、假装是犯罪嫌疑人或受害者（刑法典第 457 条）或在 证据中缺少事实依据（刑法典第 458 条）都将负刑事责任。

-Que se persona en estas Dependencias como titular de una tarjeta de crédito VISA, contratada con la entidad financiera Bankia, la cual ha tenido siempre en su poder.

被告人是 BANKIA 的 VISA 卡客户，并拥有银行卡的所有权。

-Que entre las fechas reseñadas, autor/es desconocidos han realizado un total de dieciséis cargos con la tarjeta de crédito, asociados a su cuenta de crédito número 623584561900 2948 por un importe aproximado de mil trescientos euros (1300 Euros), haciendo en este acto entrega de la copia de los movimientos reseñados.

在上述日期期间, 有人通过此卡完成了 16 笔交易, 卡号为 6235845619002948 , 并支 出约 1300 欧元，报案人提交了上述交易的详单。

-Que por tales hechos se ha personado en el día de hoy en la sucursal financiera Bankia de las inmediaciones de su domicilio, donde le han anulado la tarjeta de crédito.

由于出现上述异常, 报案人于本日去其住宅附近的 BANKIA 分行办停此卡

-Que dichos cargos se corresponden con diferentes empresas de telefonía móvil, no pudiendo precisar cómo se han realizado.

上述交易与多个移动通信公司有关，报案人不能确定交易是如何进行的。

- Que no tiene/n más que decir, firmando su declaración en prueba de conformidad, en unión del Instructor. CONSTE Y CERTIFICO. 
别无他事，双方在下方签名。警长在下面签字。

\section{Texto 2}

\section{DILIGENCIA DE INFORMACIÓN DE DERECHOS AL DETENIDO}

Nombre:

Apellido:

犯罪嫌疑人的权利

姓: 名:

A las.....horas del día... y en incumplimiento de lo dispuesto en el artículo 520 de la ley de Enjuiciamiento Criminal (L.O.14/83) se procede a poner en conocimiento del epigrafiado que ha sido detenido por su participación en un presunto delito de ....., así como los derechos que le asistan consistentes fundamentalmente en:

XX 日 XX 时 XX 分, 根据现行的刑事诉讼法（1983 年第 14 号基本法）第 520 条, 犯 罪嫌疑人 XXX 因被推定参与...行为以...罪被逮捕, 其现有基本权利如下:

1. No declarar si no quiere, no declarar contra sí mismo y no confesarse culpable, y no contestar alguna/s de las preguntas que se le formulen o manifestar que sólo declarará ante el juez.

1. 如果犯罪嫌疑人不愿意, 可不交代问题, 不自证其罪, 不认罪, 不回答警方提出的 那些只有在法庭上才能回答的问题。

2. Designar abogado y solicitar que esté presente en su declaración e intervenga en todo reconocimiento de identidad que se efectúe. En otro caso, se le designará de oficio.

2. 指定律师并申请在他交代问题时在场陪同，或为其指派官方律师。

3. Designar a la persona que desea poner en conocimiento el hecho de su detención y el lugar de custodia

3. 向指定的人告知其被逮捕的事实及羁押地点。

4. De ser extranjero, tiene derecho a que esta comunicación se realice a la Oficina Consular, y de ser asistido gratuitamente por un intérprete.

4. 如果是外国人, 有权与其领事馆进行沟通, 并免费获得译员的帮助。

5. Ser reconocido por el médico forense u otro de los facultativos que la ley establece.

5. 被法医或被法律规定的其他医生检查鉴定。

De los expresados derechos, el detenido manifiesta su deseo de:

就上述权利, 犯罪嫌疑人愿意选择:

- Ser asistido por el letrado D. 要得到律师援助。

- Ser asistido por el letrado de turno de oficio. 要得到官方律师援助。 
- Que se comunique la detención y lugar de custodia a,... 向...告知被拘捕的事实及羁押 地点。

- Que se comunique la detención al consulado. 向领事馆告知被拘捕的事实

- Ser asistido por intérprete 要求译员协助。

Leída y conforme. La firma, de lo que como Secretario CERTIFICO.

本人已阅读并确认签字, 书记员特此证明。 